\title{
NFCPlay: using NFC tags on VR mobile games to overcome the lack of input on VR Headsets
}

\author{
$1^{\text {st }}$ Ezequiel França dos Santos \\ Pontifícia Universidade Católica de São Paulo (PUC-SP) \\ ezequiel.pucsp@gmail.com \\ $3^{\text {rd }}$ José Carlos Buesso Junior \\ Pontifícia Universidade Católica de São Paulo (PUC-SP) \\ jbuesso@gmail.com
}

\author{
$2^{\text {nd }}$ Reinaldo Augusto de Oliveira Ramos \\ Pontifícia Universidade Católica de São Paulo (PUC-SP) \\ raoramos@pucsp.br
}

\begin{abstract}
User input in Virtual Reality (VR) mobile games on the touch screen have been a problem in the gaming industry for many years. Some solutions using timers, waiting to look at something to act, have been proposed and used in several games. Some VR Headsets models had a magnetic button on the side. However, this button caused interference in the magnetometer and the GPS module, essential modules for developing games using VR. This work aims to present the use of an NFC tag (Near Field Communication) as an input mechanism for the player to overcome the lack of input on VR Headsets.
\end{abstract}

Index Terms - vr input, mobile vr games, mobile input

\section{INTRODUCTION}

On the development of mobile VR games, the majority of mobile VR Headsets does not provide any input. Some techniques are applied, for example, using GPS or cameras to interact with the environment or fixed eyes for some time when they do some action.

When we put the mobile device inside the VR Headset, they also restrict their usage due to constrained input and output capabilities. One approach to compensate these constraints of mobile devices is the use of the interaction with physical objects like visual markers, QRCodes, or NFC-tags [2].

This work provides a framework upon NFC tags' technology to explore how VR mobile interaction with a simulated touch can leverage VR mobile gaming. The framework works on the newest iOS devices as well on all Android devices that support NFC technology.

\section{OBJective}

VR headsets based on Google Cardboard V1, in most cases, do not have any input. Some models have a third-party Bluetooth control, but in the majority cases, commonly, the eye positioning timing is used as an input. This work aims to present the development of a framework that made it possible the NFC technology as input on mobile VR games.

\section{Materials AND Methods}

\section{A. Near Field Communication (NFC)}

NFC is defined as a short-range remote communication that is proposed to be used on smartphones. It is based on "Radio Frequency Identification" (RFID) technology. It is a contactless framework that uses "radio frequency" (RF) waves to exchange information over a short separation [3] bidirectionally [4]. In our case, we are working in a unidirectional flow, acting just as a reader.

1) Android: Android, Google's operating system for smartphones, has been integrated with many extra features such as NFC technology (i.e., NFC-Enabled Smartphones). All NFCEnable Android smartphones can read an NFC tag in the background, making it compatible with our solution.

2) iOS: iOS is the Apple OS for the iPhone, and our project has some limitations on iOS. Apple had announced a major update for Near Field Communication (NFC) with iOS 13. All iPhones upper to iPhone 7 have now NFC-Enabled features, but only the iPhone XS, XS Max, and XR models allow users to scan NFC tags without an app using a new background tag reading feature [5] [6].

\section{B. VR Headset}

Conforming Xiangyuan Fang (2015), TVR TIME MACHINE VR COMPANY VR headsets are the kind of VR display devices that are based on the computer or mobile phone showing 3D content, usually embarking with a lens box or plastic shell [7].

After wearing VR headsets display equipment, users could appreciate 3D content.

Our project was created to VR headsets based on Google Cardboard V1 style, the most common on the market, especially in Brazil, since Google Cardboard V2, not commercialized in Brazil, V2 fixed the lack of input on VR Headsets with a conductive foam button [8].

\section{Unity $3 D$}

Unity3D is the most popular game engine in the market [9]. Unity is a cross-platform game engine developed by Unity Technologies. The engine can be used to create threedimensional, two-dimensional, virtual reality, and augmented reality games, as well as simulations and other experiences [10] [11].

We decided to use Unity since compared to other engines, Unity provides easy portability to both iOS and Android. 


\section{Development and Distribution}

The framework was created in native code for both platforms and unified in a wrapper in Unity3D. The framework generates an event-based subscribe activated by any NFC tag, which simulates a touch on an object in the game.

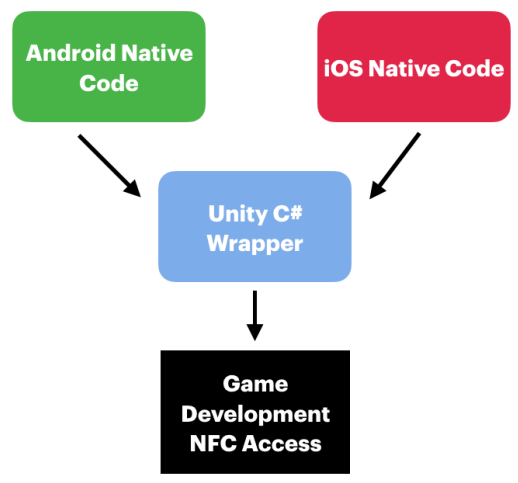

Fig. 1. Architeture of NFCFlay Framework

The Android Native code was developed in Java and iOS Native Code in Objective-C. The framework is available on Github $^{1}$, and we plan to release that on Unity Asset Store soon.

\section{RESULTS AND DisCUSSION}

We decided to use NFC since this technology, compared to other wireless communication (e.g., Bluetooth, RFID or WiFi technologies), had more advantages to our case such as: NFC does not need energy and their response is fast, moreover in a game speed is crucial because we do not want delays on our inputs, plus it has tactile feedback since all devices vibrate when an NFC tag is touched.

We created a framework using native code for iOS and Android, considering that native code delivers us an efficient utilization of the device's capabilities such as various sensors, cameras, network interfaces [12]. A photo-realism suspense game demo was also created ${ }^{2}$ alongside a "NFCPlay Logo"(Fig.2). In the future they might be distributed on Unity Assets Store, providing other developers a solution to the lack of input on VR Headsets.

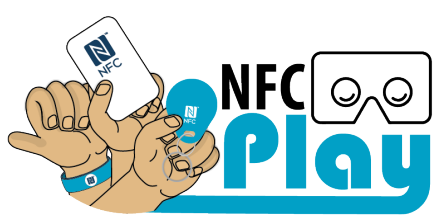

Fig. 2. NFCPlay Logo (you can use any tag, cards, wrists or key chain)

The ergonomy to play is good (Fig.3) because there are no huge movements, just a single tap of the NFC tag on the VR Headset, and the action is done.

\footnotetext{
${ }^{1}$ https://github.com/ezefranca/NFCPlay

${ }^{2} \mathrm{https} / / /$ github.com/masterrey/VRSJ
}

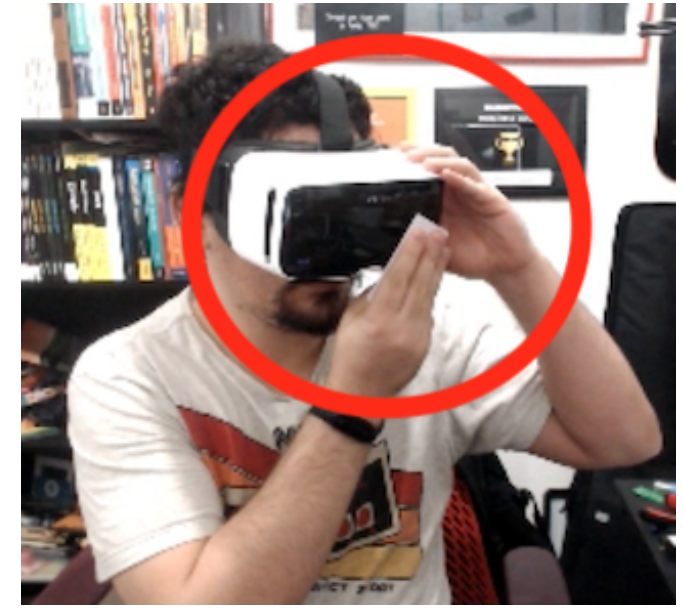

Fig. 3. Player using the NFC Card to do some action on a game

\section{CONCLUSIONS}

The NFCPlay framework was displayed as a reasonable solution to the lack of input on VR Headsets. For future work, we plan to mix visual actions with NFCPlay to create contextual menus, interactive actions, and other solutions.

\section{REFERENCES}

[1] P. C. Garrido, G. M. Miraz, I. L. Ruiz and M. Á. Gómez-Nieto, "Near Field Communication in the development of ubiquitous games", 2010 International Conference for Internet Technology and Secured Transactions, London, 2010, pp. 1-7.

[2] G. Broll, E. Rukzio, M. Paolucci, M. Wagner, A. Schmidt and H. Hussmann, "Perci: Pervasive Service Interaction with the Internet of Things," in IEEE Internet Computing, vol. 13, no. 6, pp. 74-81, Nov.Dec. 2009, doi: 10.1109/MIC.2009.120.

[3] Teh, P. L.,Ling, H. C., Cheong, S. N. (2013, December). NFC smartphone based access control system using information hiding. In 2013 IEEE Conference on Open Systems (ICOS) (pp. 13-17). IEEE.

[4] Ayu, M. A., Mantoro, T., Ismail, S. A., Zulkifli, N. S. (2012, May). Rich information service delivery to mobile users using smart posters. In 2012 Second International Conference on Digital Information and Communication Technology and it's Applications (DICTAP) (pp. 149153). IEEE

[5] Bluebite.com. 2020. iPhone NFC Compatibility. [online] Available at: ¡https://www.bluebite.com/nfc/iphone-nfc-compatibility; [Accessed 01 September 2020].

[6] Inc., Apple. "Core NFC Enhancements - WWDC 2019 - Videos." Apple Developer, ihttps://developer.apple.com/videos/play/wwdc2019/715.i [Accessed 01 September 2020].

[7] Xianyuan Fang, "Mobile Terminal VR Game Design and Development-Project Experience of Gear VR Game 'Finding,,'HighTechnology Industrialization, Nov. 2015, pp. 66-73.

[8] Arvr.google.com. 2020. Get Cardboard - Google VR. [online] Available at: ¡https://arvr.google.com/cardboard/get-cardboard/i [Accessed 01 September 2020].

[9] Messaoudi, Farouk Ksentini, Adlen Simon, Gwendal (2015). Dissecting Games Engines: the Case of Unity3D. 10.1109/NetGames.2015.7382990.

[10] Axon, Samuel (September 27, 2016). "Unity at 10: For better-or worse-game development has never been easier". Ars Technica. Archived from the original on October 5, 2018. Retrieved October 17, 2018.

[11] Takahashi, Dean (September 15, 2018). "John Riccitiello QA: How Unity CEO views Epic's Fortnite success". VentureBeat. Archived from the original on September 17, 2018. Retrieved October 17, 2018.

[12] Lim, Seung-Ho. (2015). Experimental Comparison of Hybrid and Native Applications for Mobile Systems. International Journal of Multimedia and Ubiquitous Engineering. 10. 1-12. 10.14257/ijmue.2015.10.3.01. 